\title{
Performance Updating of Concrete Structures Using Proactive Health Monitoring: A Systems Approach
}

\author{
M. Imran Rafiq \\ Department of Civil Engineering, Faculty of Engineering and Physical Sciences, University of Surrey, Guildford, \\ Surrey, GU2 7XH, UK \\ Correspondence should be addressed to M. Imran Rafiq, m_imranrafiq@hotmail.com
}

Received 27 July 2012; Accepted 8 October 2012

Academic Editors: M. Maslehuddin and G. Ranzi

Copyright () 2012 M. Imran Rafiq. This is an open access article distributed under the Creative Commons Attribution License, which permits unrestricted use, distribution, and reproduction in any medium, provided the original work is properly cited.

Uncertainties in predictive models for concrete structures performance can influence adversely the timing of management activities. A methodology has been developed that uses data obtained through proactive health monitoring to increase the confidence in predicted performance by reducing the associated uncertainties. Due to temporal and spatial variations associated with climatic changes, exposure conditions, workmanship, and concrete quality, the actual performance could vary at different locations of the member. In this respect, the use of multiple sensors may be beneficial, notwithstanding cost and other constraints. Two distinct cases are identified for which an updating methodology based on data from multiple sensors needs to be developed. In the first case the interest lies in improving the performance prediction for an entire member (or a structure) incorporating spatial and temporal effects. For this purpose, the member is divided into small zones with the assumption that a sensor can be located in each zone. In the second case, the objective is to minimise uncertainties in performance prediction, or to increase the redundancy of health monitoring systems, at critical locations. The development of updating methodologies for the abovementioned scenarios is described in this paper. Its implications on the management activities, for example, establishing the timing of principal inspections, are evaluated and discussed.

\section{Introduction}

In the UK, the Highways Agency is administering over 9,000 trunk road and motorway bridges that are valued at over $\mathfrak{E} 20$ billion. More than $65 \%$ of those are either reinforced or prestressed concrete bridges [1]. These structures represent $2 \%$ of the national network length but $30 \%$ of its total asset value. The effective maintenance management of these high value assets is of increasing importance and significant research is directed towards this area. In the UK, $50 \%$ of the total bridge and large culvert stock were constructed between 1960 and 1980 [2]. In most developed countries with already established, but aging, infrastructure, the investment on maintenance of these structures is either approaching, or has already exceeded, the capital spent for new construction. For example, the UK's Highways Agency supports a maintenance program of $\mathfrak{E} 7$ billion from 2001 to 2010 for their administered transport network [3].
Visual inspections are widely used to aid maintenance management of almost all deterioration prone systems. Despite obvious benefits (e.g., simplicity, cost, and access to $100 \%$ of the visible surface), they are intermittent, subjective, and applicable only to exposed surfaces, and the need for access may involve disruption to normal operations. Further, the extent of damage cannot be estimated only by a surface examination; the signs of deterioration may not appear at the surface until it has caused severe internal damage, especially if the defect is not at, or close to, the surface. Furthermore, visual examinations can only provide qualitative information with respect to condition which may not relate explicitly or fully to safety.

Nondestructive examination (NDE) addresses several of these drawbacks and has gained popularity among the maintenance and management communities. The need to be in close vicinity of the area to be inspected is a major hurdle in its use for several applications, for example, bridges where 
it becomes costly primarily due to the indirect costs of bridge closure and traffic management. The "direct" and "indirect" data provided by NDE can often be related explicitly to safety levels but they are generally intermittent in both time and space.

In recent years, health monitoring methods are being developed as a continuation of the research into NDE methods. These range from simple methods (such as halfcell measurements) to more sophisticated technology such as corrosion risk sensors, possibly with remote sensing capability. These structural health monitoring (SHM) methods can provide real-time information on system deterioration characteristics, for example, Daly and Watts [4] and CIRIA [5], which can be used effectively for maintenance planning. There are, however, limitations and issues associated with these methods; for example, the information is limited to specific locations at which the sensors are installed, the accuracy is limited depending on the sensor type being used and the quantity being monitored, and costs can be higher compared to other methods. Some other issues that must be addressed to facilitate the effective use of, and gain full benefits from, SHM include optimum number of instrumentation locations, nature of data obtained (i.e., discrete versus continuous) and their interpretation (i.e., relation between the quantity being monitored and the system property under consideration), and methods to handle erroneous and/or unexpected output.

With regard to the spatial variability of deterioration processes, a major concern in the use of the above systems is that they only provide information at a small number of specific locations; careful thought has to be given as to how these results can be considered as representative (or not) for the element or entire system.

Unexpected results also pose many questions. If, for example, several sensors are installed at various locations in a system what should the conclusion be, if a sensor contradicts a similar closely located sensor? Alternatively, what if a sensor close to a known defect indicates a less severe condition, whereas another at some distance away indicates a worse condition, and so forth. Other possibilities include the absence of, or clearly erroneous, readings obtained from a particular sensor. But even when the sensors are free from obvious errors how confident should we be regarding their output, and to what extent can this information be used for performance prediction purposes?

The frequency of measurements required on a system depends primarily on the phenomenon being monitored; for example, the frequency of information obtained through sensors should be very high for live load measurements on a structure to avoid any important reading being missed out. On the other hand, if the phenomenon being monitored is corrosion in a concrete structure, the sensors, output need not be that frequent. Even though the process of health monitoring may be continuous in nature, the output from the sensors could either be of a continuous or a discrete form depending on the parameters being monitored and the type of sensors being used.

In a distinct strand of research, deterioration models have been developed to estimate and predict the performance of structures. Research on different materials, for example, concrete, steel, and masonry, in the area of performance prediction has lead to the development of predictive models for a range of conditions, to a varying degree of complexity. These models have the potential to provide information on materials' deterioration, which can be continuous in time and space. The downside of the models is that uncertainties associated with the nature and rate of deterioration, the demand (past, present, and future), and other factors which influence the actual performance of these structures are considerable and subject to change during their service life. These uncertainties can be treated formally using probabilistic methods and an increasing shift towards probabilistic deterioration modelling is evident in the literature. The uncertainties in the deterioration modelling process can be reduced by introducing additional information, through a range of inspection and monitoring procedures carried out at different stages of the structure's life, resulting in increased confidence in the predicted performance.

A methodology has been developed by the authors [6] that uses data obtained through proactive health monitoring to increase the confidence in predicted performance by reducing the associated uncertainties. Due to temporal and spatial variations associated with climatic changes, exposure conditions, workmanship, and concrete quality in a bridge, the actual performance could vary from member to member or even at different locations of the same member. In this respect, the use of multiple sensors may be beneficial, notwithstanding cost and other constraints.

This paper describes the development of updating procedures incorporating information from multiple sensors with a view of predicting the performance of the monitored domain with increased confidence. These are demonstrated through application on concrete structures prone to chloride-induced deterioration.

\section{Deterioration Modelling for RC Structures}

In general, deterioration of concrete structures is associated with the corrosion of reinforcement embedded in concrete [7]. This is caused mainly by either carbonation or chloride attack. These mechanisms are unique in the sense that the aggressive agents penetrate into the concrete without any visual signs of deterioration until they reach the reinforcement level and initiate corrosion. In addition to the loss of section in the steel bars, the expansive products of corrosion cause delamination and spalling of concrete, which ultimately may lead to failure of the structure. Chloride ions have been found to be a major factor contributing towards deterioration in reinforced concrete structures [7]. Hence, this study will focus primarily on this aspect of the deterioration, though the procedures can be generalised to address other deterioration mechanisms.

Generally aggressive agents penetrate from outside sources; for example, the amount of chloride cast into concrete is limited by design codes, but the problem starts as chloride ions ingress from outside. Whatever the source (deicing salts, marine environment), the ingress is a complex 
phenomenon and involves different transport processes, for example, initial penetration, absorption and capillary suction, and diffusion. The deterioration processes in concrete have been modelled using a variety of approaches, for example, based on Fick's 2nd law of diffusion [8], based on Markov chain process [9], using neural network models [10], modified solution of Fick's law assuming a fixed amount of surface contents [11], modelling diffusion using Nernst-Einstein equation [12], experimental data of cracked concrete [13], and diffusion as a combination of Knudsen and Viscous flow [14]. The models range from empirical (based entirely on experimental results) to scientific (based entirely on scientific principles and physical laws) including a wide range of semiempirical models (based on simplified scientific models that are calibrated through laboratory or field experiments). Several benchmark studies have been carried out to standardize the chloride ingress models, for example, HETEK [15] and Duracrete [16] but consensus regarding any particular phenomenon to be used for modelling has not yet emerged. Overall, the majority of researchers are using Fick's diffusion law as a representative phenomenological model. The fact that some of the parameters in deterioration modelling are time dependent (e.g., [17]) adds complexity to the modelling process.

Uncertainty in the variables involved in the deterioration process is generally modelled using random variables. Spatial variability of the deterioration process and lack of knowledge regarding its details also contribute towards the complexity in modelling deterioration and associated uncertainty (e.g., [18]). The amount of uncertainty in chloride-induced deterioration is significant and limits the applicability of the predictive models to short to medium range predictions.

A typical model for the time to corrosion initiation based on Fick's second law of diffusion is presented:

$$
T_{I}=\frac{E_{\bmod } X^{2}}{4 D\left[\operatorname{erfc}^{-1}\left(C_{\mathrm{th}} / C_{o}\right)\right]^{2}},
$$

where $T_{I}$ is the time to corrosion initiation at any given depth $X ; D, C_{o}, C_{\text {th }}$, and $E_{\text {mod }}$ represent the effective diffusion coefficient, surface chloride concentration, threshold chloride concentrations, and model uncertainty factor, respectively. Due to uncertainties in the quantification of these parameters, a probabilistic approach for deterioration modelling is generally adopted [19]. A similar approach was adopted by Rafiq et al. [6] resulting in a typical distribution for the corrosion initiation time as shown in Figure 1.

This curve can be interpreted in two different ways. The ordinate gives the probability that corrosion initiation at rebar level will be reached up to any particular point in time (abscissa). If an acceptable (tolerable) target probability can be specified, the curve could be used to estimate the point in time at which certain management actions are to be taken (e.g., if a target probability of 0.3 is considered, actions such as principal inspections would be needed after 10 years). On the other hand, assuming spatially uniform conditions, the ordinate may be interpreted as the fraction of the area of a member exhibiting corrosion activity normalized by the total area. In this case, the target (or threshold) would represent

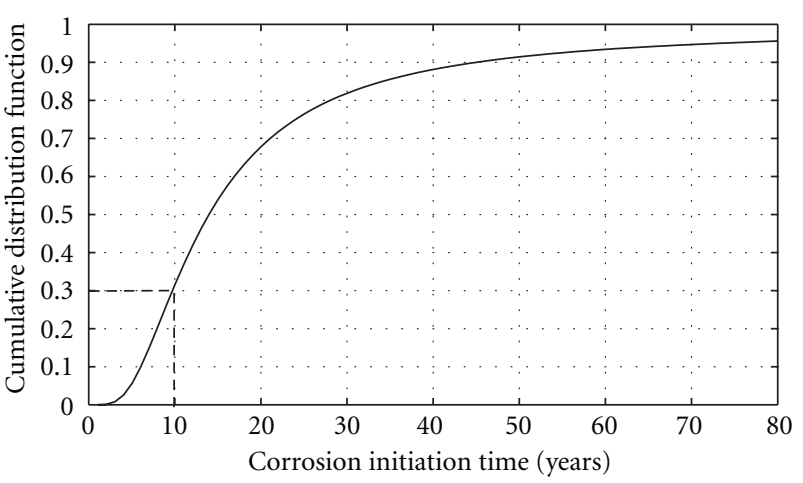

FIGURE 1: Distribution for the corrosion initiation time.

the maximum corrosion damage tolerated for any particular member or structure.

\section{Monitoring of Corrosion in Concrete Structures}

The mechanism of corrosion may be split into two phases, "initiation" and "propagation" [20]. From the point of view of the management of structures, the maintenance becomes very costly once the corrosion reaches the second phase; hence, the focus of this paper will mainly be on the initiation phase of corrosion. The methodology developed can easily be extended to the propagation phase, where this is merited.

During the initiation phase, the corrosion risk of a reinforced concrete structure can be monitored through either chloride content measurements, or by measuring the penetration of the threshold chloride contents, in the cover concrete. Chloride measurement probes have been developed though they appear to still be in the testing and validation stage. Corrosion risk probes have also been developed, and instruments available for this include the following:

(i) Ladder Arrangement (Figure 2);

(ii) Metallic Nail System;

(iii) Expansion Ring System (Figure 3).

The ladder arrangement can be installed in new structures or during repair works in existing structures. Expansion ring and Metallic nail systems can also be installed into existing structures without damaging the existing concrete cover. The working principle for all three systems is identical. Small pieces of steel are installed at various known depths into the cover concrete and the corrosion activity of these pieces is monitored. Corrosion initiation at these steel pieces gives an indication of the corrosion penetration depth into the cover concrete. A curve of the corrosion penetration depth is plotted against time, which can then be extrapolated to predict the time to corrosion initiation at the rebar level. It is worth mentioning that these instruments are still at their infancy but have delivered promising results in laboratory conditions. These are being tested on actual structures but 


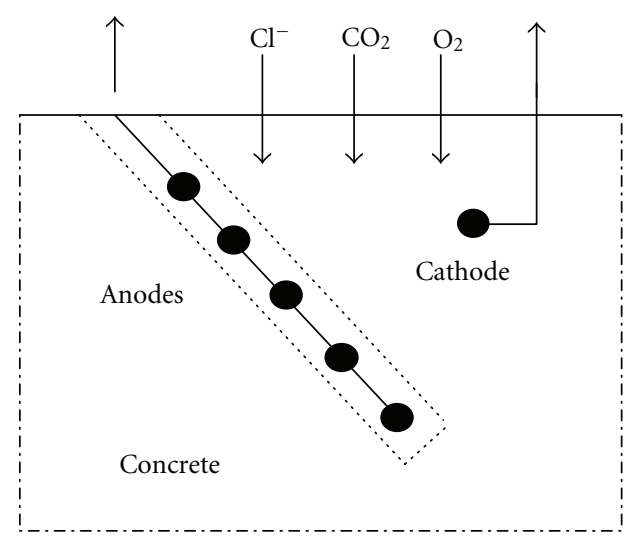

(a)

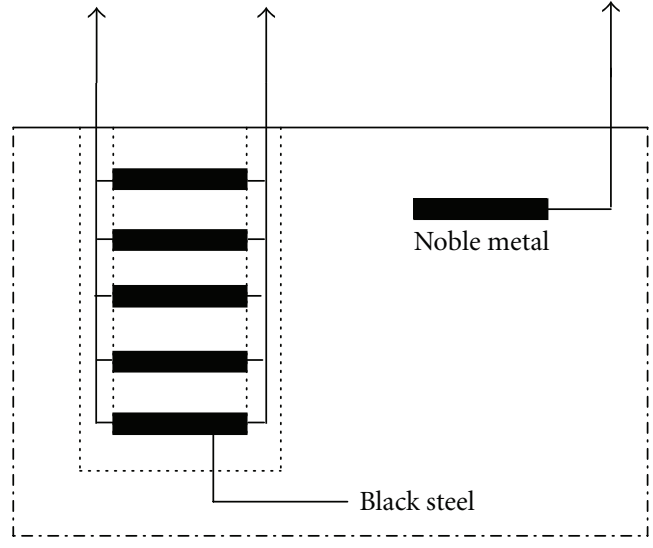

(b)

FIGURE 2: Ladder arrangement [21].

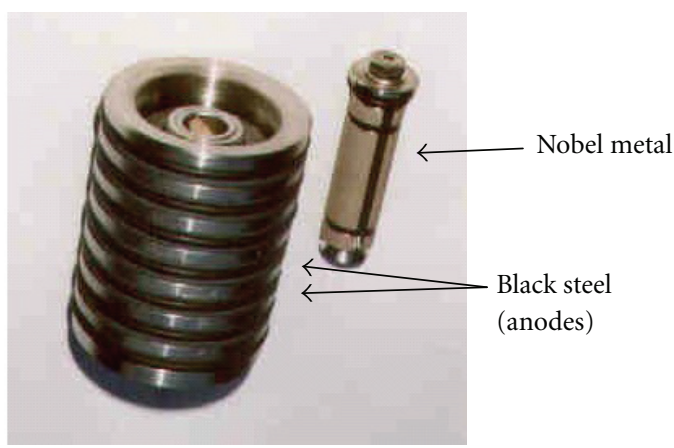

FIGURE 3: Expansion Ring System [22].

very little is available in the current literature about their effectiveness in the field conditions.

\section{SHM Supported Deterioration Prediction}

Limitations in the use of predictive models and SHM methods in predicting future performances have been highlighted in the previous sections. There are clear benefits in combining the information from the two sources. However, this information is often diverse in quality and quantity and is subject to various uncertainty sources. Thus, a primary objective of combining prior information with monitoring data should be to combine uncertainties, whilst realising that there are different and diverse sources.

A powerful and versatile approach dealing with performance evaluation and prediction of systems in the presence of uncertainty is the Bayesian approach. This approach has had a significant impact in nuclear plants assessment and in the evaluation of healthcare system. More recently, similar techniques have been used successfully in offshore installations and steel bridges, for the planning and optimization of inspection and maintenance schedules [23-27]. The Bayesian updating approach can be used to incorporate information obtained from different sources at different point-in-time during long service lives, for example, either from detailed inspections and monitoring or even from the qualitative assessment methods, that is, visual inspections or service records, and so forth. An application related to concrete structures is presented by Faber and Sorensen [28], where inspection results are used to evaluate the condition states of bridges at any given time.

A framework has been proposed that combines the information obtained from SHM with deterioration modelling to improve the confidence in the predicted performance [6]. A sensitivity study of various input models on the performance prediction concluded that the effects of uncertainty in various input models are considerably reduced by such updating [29].

Let the probability distribution for the "prior time to failure" at location $X_{C}$ (i.e., cover depth in a concrete member) be

$$
F_{T}^{\prime}(t)=P\left[T\left(X=X_{C}\right) \leq t\right] .
$$

It represents the probability that the "time to failure" at a given location $X_{C}, T\left(X=X_{C}\right)$, is less than or equal to any given time, $t$. Assuming, for simplicity, that the sensor output is discrete, two updating scenarios are possible. The first scenario is the case when the health monitoring system confirms that the predefined limit state has not been attained at the sensor location (confirmation of "safety") at a particular point in time (i.e., at the time of monitoring, $\left.t_{m}\right)$; the "actual time to failure," $t_{i}$, of the sensor (located at $X_{i}$ ) is not known but is greater than the time of monitoring, that is, $t_{i}>t_{m}$. Hence, the 'predicted time to failure at sensor location, $T\left(X=X_{i}\right)$, should be greater than $t_{m}$. This information can be used to update the distribution for time to failure using

$$
F_{T}^{\prime \prime}(t)=P\left[T\left(X=X_{C}\right) \leq t \mid T\left(X=X_{i}\right)>t_{m}\right] .
$$

When the health monitoring system confirms the attainment of a limit state at the sensor location (confirmation of "failure") at a given time (i.e., second updating scenario), the "time of failure" at the sensor location would be equal to 
the time of attainment of the limit state, that is, $T(X=$ $\left.X_{i}\right)=t_{i}=t_{m}$. In order to account for various instrument/measurement uncertainties, it is assumed that a sensor cannot be perfect. Instead of yielding the "exact time to failure" at the sensor location, two limiting values for the "time to failure" are introduced so that below the lower time limit failure has not occurred and above the upper limit failure has occurred. This can mathematically be expressed as " $T\left(X=X_{i}\right) \leq t_{i}$ and $T\left(X=X_{i}\right)>t_{i}-t_{\text {ins," }}$ " that is, a sensor confirms the attainment of limit state at the time $t_{i}$, whereas it would have not attained the limit state at the time $t_{i}-t_{\text {ins }}$. Here the time interval, $t_{\text {ins }}$, accounts for the uncertainty in instrument and measurement method used. Higher value of the time, $t_{\text {ins }}$, would reflect higher instrument/measurement uncertainty and would reduce the confidence in the posterior predicted performance and vice versa. The posterior distribution for time to failure for this case is given by the following.

$$
\begin{aligned}
& F_{T}^{\prime \prime}(t)=P[ T\left(X=X_{C}\right) \leq t \mid T\left(X=X_{i}\right) \\
&\left.\leq t_{i} \cap T\left(X=X_{i}\right)>\left(t_{i}-t_{\text {ins }}\right)\right] .
\end{aligned}
$$

Combining the two scenarios and using Bayesian event updating framework, the posterior distribution for the "time to failure" for a total of " $n$ " sensors would become [6]

$$
\begin{aligned}
& F_{T}^{\prime \prime}(t) \\
& \qquad=\frac{P\left[\left[T_{I}\left(X=X_{c}\right) \leq t\right] \bigcap_{i=1}^{n}\left[M_{i} \leq 0\right] \bigcap_{i=1}^{n}\left[M\left(X_{i}\right)>0\right]\right]}{P\left[\bigcap_{i=1}^{n}\left[M_{i} \leq 0\right] \bigcap_{i=1}^{n}\left[M\left(X_{i}\right)>0\right]\right]},
\end{aligned}
$$

where $F_{T}^{\prime \prime}(t)$ is the posterior cumulative distribution function for the "time to failure." $X_{i}$ is the location of sensor no. $i$. $T\left(X=X_{i}\right)$ is the prior predicted "time to failure" at location $X_{i} . M\left(X_{i}\right)$ is the safety margin for expected "time to failure" at $X_{i}$ at a given time $t_{m} . M\left(X_{i}\right)=T\left(X=X_{i}\right)-t_{m}$, when "safety" is confirmed at location $X_{i} . M\left(X_{i}\right)=T\left(X=X_{i}\right)-$ $\left(t_{i}-t_{\text {ins }}\right)$ when "failure" is confirmed at location $X_{i}$ and the "time to failure" of sensor $i$, $t_{i}$, becomes known. $M_{i}$ is the Safety margin between predicted and actual "time to failure," when the "time to failure" of sensor $i$ becomes known. $M_{i}=$ $T\left(X=X_{i}\right)-t_{i}$ and $M_{i}=0$ for the "safety" confirmation case. $t_{i}=a$ is the deterministic parameter representing the time at which "failure" is detected by the sensor $i . t_{\text {ins }}=a$ is the deterministic parameter representing the time interval between the two events, that is, "confirmation of failure" and "confirmation of safety" that accounts for monitoring instruments uncertainty.

\section{Systems Updating Approach}

An inherent assumption in the above methodology is that there is only need to consider one location at which both prior and posterior (i.e., using monitored data) distributions are considered. In practice, the extent of deterioration varies considerably from one location to another. These variations can be attributed to the temporal and spatial effects of different variables involved in the deterioration process,

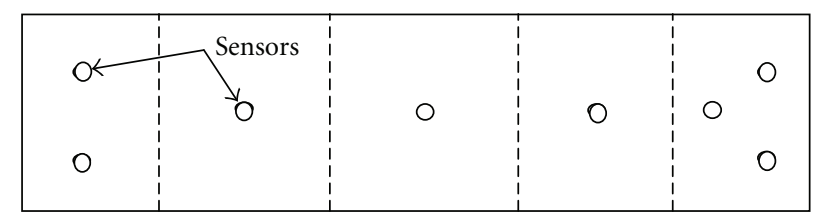

FIgURE 4: A structural member divided into five zones.

within the element and/or for different elements of a system. The actual performance in such cases could be different for different elements of a system and even at different locations of the same element. In order to explore the application of the Bayesian methodology in cases where temporal and spatial influences are present, it is assumed that the monitored domain can be subdivided into a number of smaller zones with the possibility of installing sensors within each zone. The distance between the sensors, and hence the physical size of the zone, should be large enough to avoid any spatial correlation on sensor outputs. On the other hand, the zone should be small enough to justify the assumption of uniform performance over its entire physical size.

Multiple sensors may be deployed in another scenario, that is, to increase confidence in the performance prediction of a critical location, or when a robust/redundant monitoring system is required due to critical nature of a zone.

The two cases could exist in combination, for example, a bridge deck, as shown schematically in Figure 4 . The deck is divided into several zones with a sensor located in each zone (the former case). Additional sensors may be located at high shear and moment zones (the later case).

The updating procedure to account for data from multiple sensors needs to be developed for each of the two cases bearing in mind the nature of the decision that needs to be considered. In the former case (multiple sensors in different zones), the interest is to determine updated predicted performance for the entire system under consideration. In the later case (multiple sensors in the same zone), the interest lies in improving the confidence in prediction of performance within the individual zone, or to increase the redundancy of the monitoring system at critical locations.

\subsection{Performance Updating through Sensors in Different Zones.} Consider a system divided into a number of small zones and a sensor located in each zone. The outcome of health monitoring in this case would be the "actual time to failure" at certain depth of each zone. The difference between "times to failure" at certain depth in each of these zones could reflect the spatial variation of deterioration phenomenon (due to variation in microclimate, environmental characteristics or material properties, etc.). In this case, the "time to failure" at sensor location, $t_{i}$ in (3) and (4), becomes a random variable, $T_{i}$, which can be represented by an empirical distribution using the data obtained from multiple sensors located along the space. Treating the "time to failure" at each zone as a realisation of $T_{i}$, (4) can be used to establish the distribution for time to failure of each zone. 
TABLE 1: Distribution type and values of its parameters for basic random variables.

\begin{tabular}{lcccc}
\hline Parameter & Mean & C.O.V. & Distribution & Reference \\
\hline$C_{o}$ & $3.5 \mathrm{Kg} / \mathrm{m}^{3}$ & 0.5 & Lognormal & Vu and Stewart [30] \\
$D$ (nominal) & $5 \times 10^{-5} \mathrm{~m}^{2} / \mathrm{yr}$ & & & \\
Model error $(D)$ & 1.0 & 0.2 & Normal & Vu and Stewart [30] \\
$C_{\text {th }}$ & $0.9 \mathrm{Kg} / \mathrm{m}^{3}$ & 0.19 & Uniform $\left(0.6-1.2 \mathrm{Kg} / \mathrm{m}^{3}\right)$ & Vu and Stewart [30] \\
$E_{\text {mod }}$ & 1.0 & 0.2 & Lognormal & Lentz et al. [31] \\
$X$ & $40 \mathrm{~mm}$ & 0.1 & Normal & Chryssanthopoulos and Sterrit [32] \\
$X_{i}$ & 10,20, and $30 \mathrm{~mm}$ (fully correlated) & $\sigma=1 \mathrm{~mm}$ & Normal & \\
$t_{\text {ins }}$ & 0.1 years & & Deterministic & \\
\hline
\end{tabular}

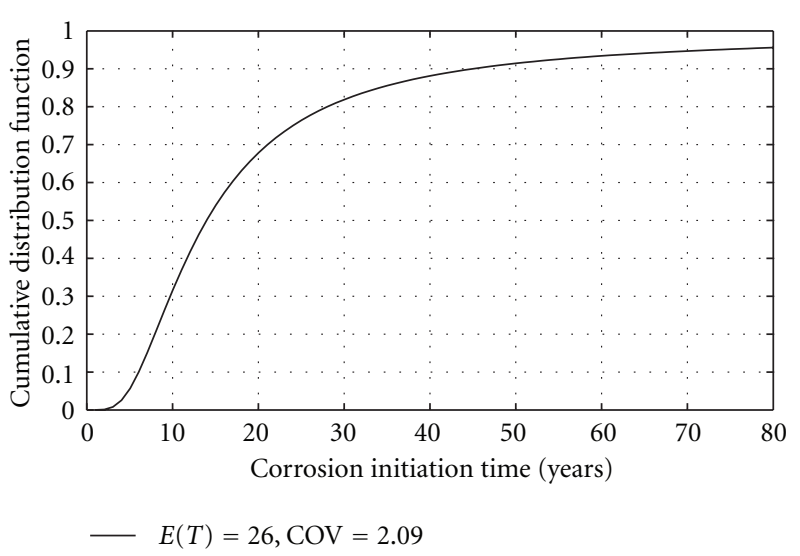

(a)

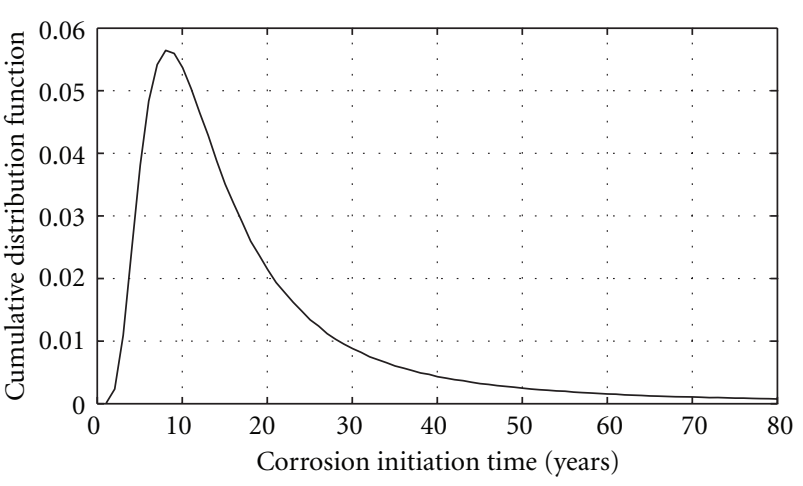

(b)

FIgURe 5: Prior corrosion initiation time at rebar level.

The distribution for posterior "time to failure" of the system, $F_{T}^{\prime \prime \text { sys }}$, (composed of different zones) can be given by

$$
F_{T}^{\prime \prime \text { sys }}(t)=\sum_{N=1}^{i} F_{T}^{\prime \prime Z i}(t) \cdot P_{T_{i}}\left(t_{i}\right),
$$

where $F_{T}^{\prime \prime Z i}(t)$ is the posterior distribution for the "time to failure" for zone $i$, and $P_{T_{i}} T(t)$ is the probability mass function for actual "time to failure" at the sensor location.

5.2. Performance Updating through Sensors in the Same Zone. In this case the objective is to either increase robustness of health monitoring instruments or to increase the confidence in prediction depending upon the critical nature of the zone under consideration. Hence, the sensors would be located relatively close to each other (within the same zone) and the assumption is that the deterioration would be uniform within that zone.

Bayesian updating can thus be applied for multiple sensors to improve confidence regarding prediction of performance of the zone under consideration. The posterior distribution for the "time to failure" using data from first sensor (that can be selected at random as the sequence is irrelevant) will be obtained from (4). This distribution will serve as a prior for the next updating sequence. The predicted "time to failure" at sensor locations, $T(X=$
$X_{i}$ ), is recalculated after each updating step. The updating is repeated until the information from all the sensors is incorporated.

The above methodologies are explored in detail through a case study on a concrete member subjected to chlorideinduced deterioration.

\section{Case Study}

In this study the system updating methodology is applied to a bridge element prone to chloride-induced deterioration. In order to demonstrate the performance updating methodology for sensors in different zones, the element is hypothetically divided into five zones with a corrosion risk sensor located in each zone. For the purpose of demonstrating the updating methodology for multiple sensors installed within the same zone, three sensors are assumed within the same zone. The results for the two cases are presented separately in this paper to explore their characteristics.

Table 1 summarises the models used for input parameters of the deterioration model. Monte Carlo simulations with Latin hypercube sampling have been used to obtain prior and posterior distributions.

Using these variables and the performance equation (1) the prior time to corrosion initiation at rebar level (nominally $40 \mathrm{~mm}$ ) is shown in Figure 5 (probability density 


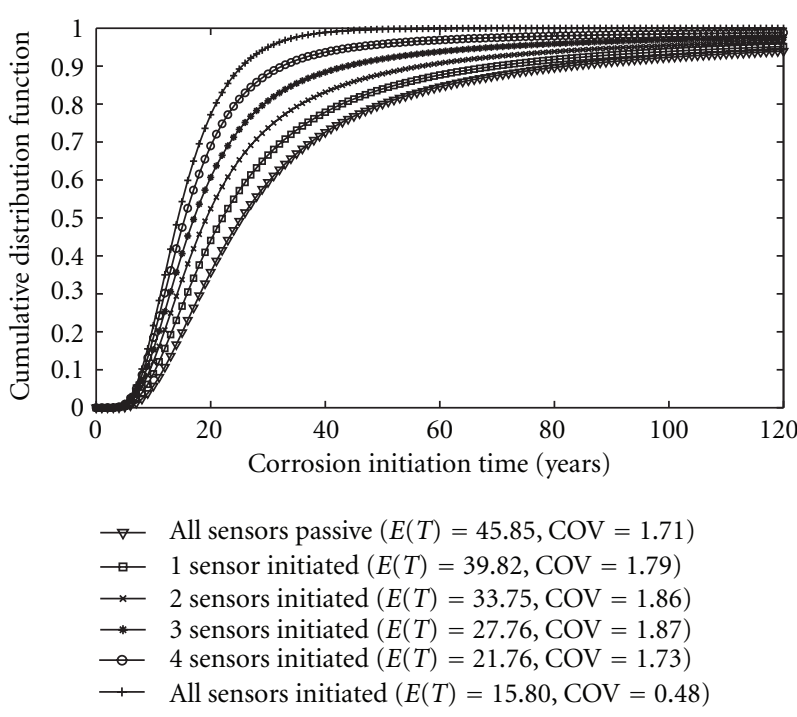

(a)

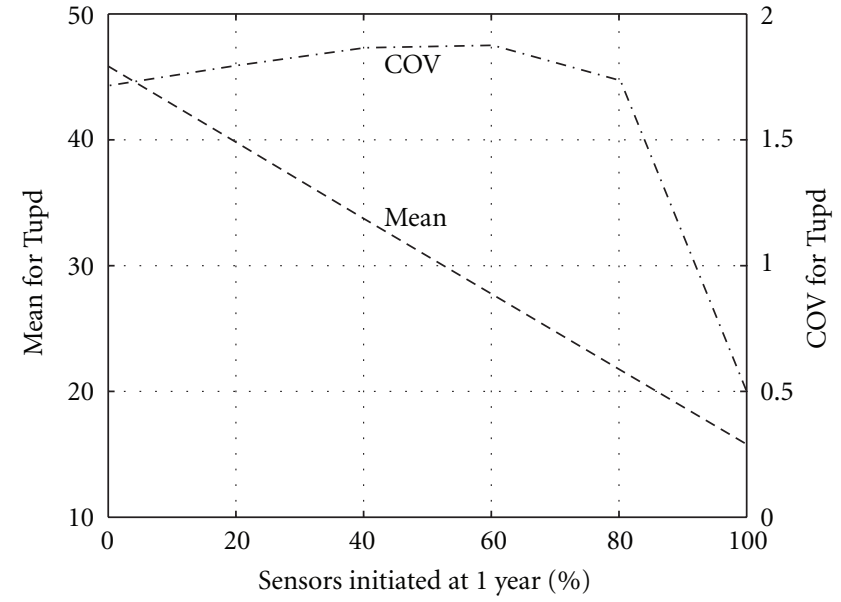

(b)

FIgURE 6: Corrosion initiation time at rebar level for different percentages of sensors showing initiation (at $10 \mathrm{~mm}$ depth).

and distribution functions). It can be seen that the mean time to corrosion initiation is 26 years with the coefficient of variation, COV, (representing the uncertainty in the prediction of corrosion initiation time) being 2.09. The variation of the mean and COV after updating for various scenarios of different number of sensors, and for different hypothesized sensor outputs, is presented in the following sections.

6.1. Updating of Performance Prediction through Sensors in Different Zones. The posterior predicted performance (corrosion initiation times) assuming five sensors (at $10 \mathrm{~mm}$ cover depth) distributed along the plan (i.e., member divided into five zones) is shown in Figure 6. The scenario is that the number of sensors (located at $10 \mathrm{~mm}$ depth) indicating corrosion initiation at 1.0 year varies from zero to five.

It is clear from the figure that if all the sensors show the same output, that is, either corrosion initiation or passivity confirmation at a given point in time, the uncertainty associated with predicted performance is considerably less than in the case where even one sensor shows opposing results.

It can also be seen that uncertainty levels for the case when all sensors show corrosion initiation $(\mathrm{COV}=0.48)$ is considerably less than the case when passivity is confirmed at the sensors $(\mathrm{COV}=1.71)$. This is because the quality of information available with the initiation confirmation case being higher (i.e., the time to corrosion initiation at the sensor location becomes available) than the passivity confirmation case (i.e., the time to corrosion initiation at the sensor location is larger than the time of monitoring, but is still an unknown quantity).

Assuming that a management action (such as a principal inspection) will be required when the probability of corrosion initiation reaches 0.3 , the time for next inspection, based entirely on the prior predictive model, is 10 years. It can be seen from Figure 6 that, depending upon the performance of a bridge and variability of the sensor outputs, the time for next principal inspection for the considered case study may vary from 14 to 19 years. In such a case, the delay in principal inspection due to improvement in performance prediction, coupled with the delay in direct and indirect management costs (e.g., repair and traffic management costs, etc.) could lead to significant whole life cost savings.

6.2. Updating of Performance Prediction through Sensors in the Same Zone. The results (posterior corrosion initiation time) assuming one, two or three sensors in the same zone are shown in Figure 7. The updating is carried out at 1.0 year, assuming all the three sensors are confirming passivity at that point in time.

It is clear that increasing the number of sensors in the same zone would increase the confidence regarding the prediction of performance (as COV for the corrosion initiation time is reducing continuously). Similar to the previous case, assuming the limiting value for the probability of corrosion initiation as 0.3 , the timing for a management action (i.e., a Principal Inspection) can be evaluated. It can be seen from Figure 7 that if passivity is confirmed after 1.0 year, the time for next inspection for this case has been delayed from 10 years (obtained from the prior case) to 19, 24, or 28 years, respectively, for one, two, or three sensors being used in the same zone.

A similar trend can be seen for the case where the sensors show initiation of corrosion at year 1.0 (Figure 8). The possibility of sensors having some probability of not functioning properly (and hence giving a "wrong" indication of passivity or a "wrong" indication of corrosion initiation) has not been included in the above results. 


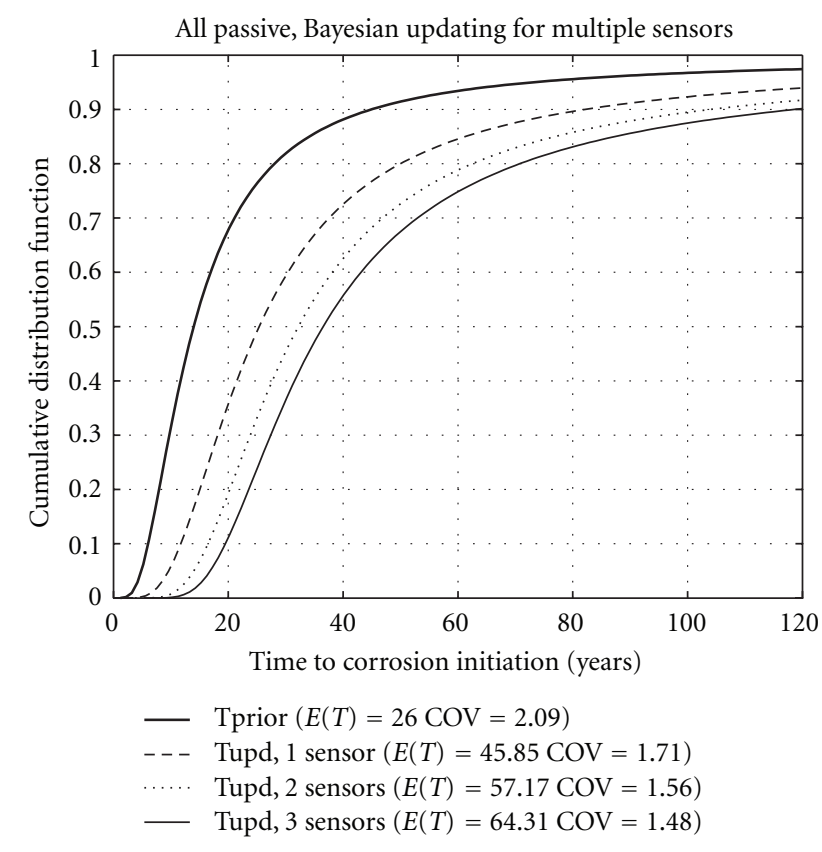

(a)

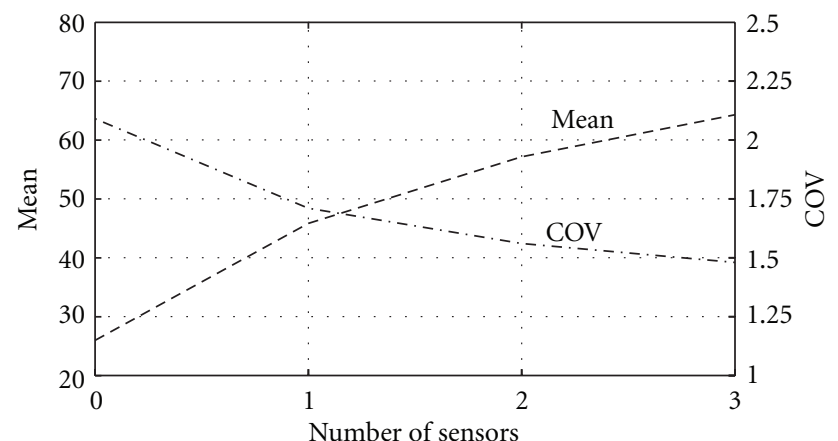

(b)

FIgURE 7: Effects of Bayesian updating for multiple sensors along the plan showing passivity confirmation.

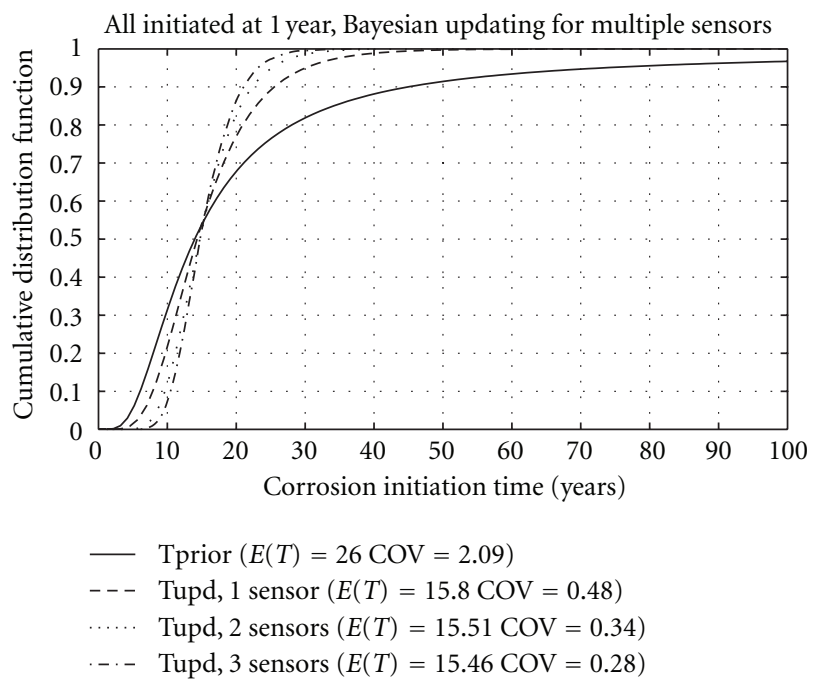

(a)

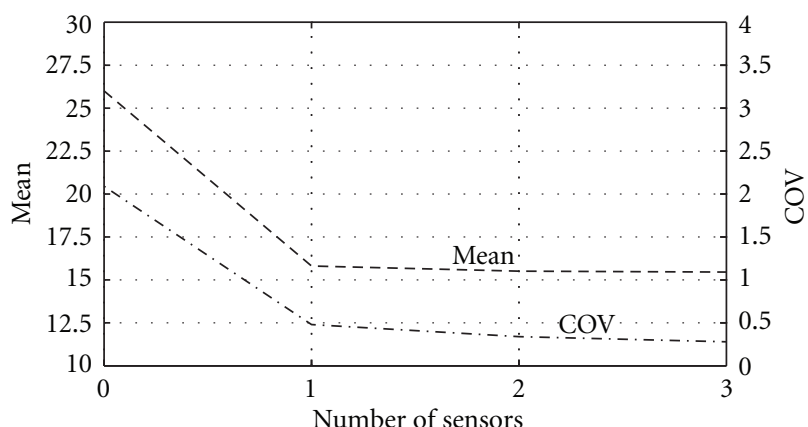

(b)

FIGURE 8: Effects of Bayesian updating for multiple sensors along the plan for initiation confirmation.

\section{Conclusions}

Uncertainties in the performance prediction of infrastructure facilities have a profound effect on the management decisions such as the timing of inspections and repairs. These uncertainties can be considerably reduced by effectively integrating the data obtained through health monitoring systems into the assessment and prediction procedures.

Due to the spatial effects of deterioration and/or to increase the redundancy of the monitoring systems, multiple sensors are required at various locations of the system. An approach for combining data from such multiple sensors is outlined in this paper. Two cases of updating have been identified and results for both of these have been summarized and discussed. It has been shown that the performance of a monitored domain representing a member, a structure, or an entire system can be updated using the proposed Bayesian procedures. Their application for chloride-induced deterioration in bridges has shown their effectiveness in reducing the associated uncertainties or in obtaining overall 
performance prediction of the member/structure by rationally combining similar data obtained through sensors at different locations. These methodologies can be used as a tool in the reliability-based management of structural systems to gain confidence in performance predictions and hence in the optimization of management activities such as repairs, maintenance, and inspections. With regard to the type of data being obtained (discrete versus continuous), it has been shown that continuous data can be converted to discrete form using limit state functions for simple applications. It is acknowledged here that the quality of information (obtained from the SHM) has a direct impact on the updated distribution; hence, the full potential of continuous data may not be apparent by using these procedures. The methodology needs to be refined and developed for such cases and this is the subject of ongoing research.

More research is also needed in other related aspects, for example, to establish the optimum number of sensors required and their distribution within the system to maximize the effectiveness of the health monitoring systems, and the development of procedures to incorporate/handle misinterpreted data and unexpected results.

Similarly, the methods for effective integration of the predicted performance of individual zones, and of the entire structure, into bridge management systems (i.e., optimization of inspection, maintenance and repair activities, etc.) need to be further developed/refined. The approach detailed herein would also benefit from being tested in the field.

\section{Acknowledgments}

The author would like to acknowledge Professor Marios Chryssanthopoulos at the University of Surrey and Professor Toula Onoufriou from Cyprus University of Technology for their unconditional support and advice during the course of this project. Their support has been vital for the success of this project and of course for the completion of his Ph.D. in this area. The author would also like to acknowledge the funding provided by the ORSAS and URS Scholarships to fund this study as part of his Ph.D.

\section{References}

[1] B. Mahut and R. J. Woodward, "Comparison of bridge management practice in England and France," in Bridge Management, G. A. R. Parke and P. Disney, Eds., vol. 5, pp. 163-170, Thomas Telford, London, UK, 2005.

[2] B. Allison and R. J. Woodward, "Inspection, testing, and monitoring of trunk road bridges in England," in Bridge Management, G. A. R. Parke and P. Disney, Eds., vol. 5, pp. 171-178, Thomas Telford, London, UK, 2005.

[3] Highways Agency, "Strategic Roads 2010: highways agency 10 years national road strategy," Highways Agency, London, UK, 2005, http://www.highways.gov.uk/.

[4] A. F. Daly and G. R. A. Watts, "High-Tech remote monitoring for the management of highway structures," Project Report PPR197, TRL Limited, 2006.

[5] N. R. Buenfeld, R. D. Davies, A. Karami, and A. L. Gilbertson, Eds., "Intelligent monitoring of concrete structures," Tech. Rep. C661, CIRIA, London, UK, 2008.
[6] M. I. Rafiq, M. K. Chryssanthopoulos, and T. Onoufriou, "Performance updating of concrete bridges using proactive health monitoring methods," Reliability Engineering and System Safety, vol. 86, no. 3, pp. 247-256, 2004.

[7] G. C. M. Gaal, C. V. D. Veen, and M. H. Djorai, "Deterioration of concrete bridge in the Netherlands," in Structural Faults and Repairs, M. C. Forde, Ed., Engineering Technics Press, Edinburgh, UK, 2001.

[8] M. Collipardi, A. Marcialis, and R. Turriziani, "The kinetics of penetration of chloride ions in concrete," II Cemento, vol. 4, pp. 157-164, 1970.

[9] M. Cesare, J. Santamarina, C. Turkstra, and E. Vanmarcke, "Modelling bridge deterioration with Markov Chains," Journal of Transportation Engineering, vol. 118, no. 6, pp. 820-833, 1992.

[10] M. F. Elkordy, K. C. Chang, and G. C. Lee, "Neural networks trained by analytically simulated damage states," Journal of Computing in Civil Engineering, vol. 7, no. 2, pp. 130-145, 1993.

[11] D. M. Frangopol, K.-Y. Lin, and A. C. Estes, "Reliability of reinforced concrete girders under corrosion attack," Journal of Structural Engineering, vol. 123, no. 3, pp. 286-297, 1997.

[12] S. Chatterji, "Transportation of ions through cement based materials. Part 1 fundamental equations and basic measurement techniques," Cement and Concrete Research, vol. 24, no. 5, pp. 907-912, 1994.

[13] C. Q. Li, "Initiation of chloride-induced reinforcement corrosion in concrete structural members-prediction," ACI Structural Journal, vol. 99, no. 2, pp. 133-141, 2002.

[14] C. Q. Li, J. J. Zheng, and L. Shao, "New solution for prediction of chloride ingress in reinforced concrete flexural members," ACI Materials Journal, vol. 100, no. 4, pp. 319-325, 2003.

[15] HETEK, "Chloride penetration into concrete," State of the Art report no. 53, The Danish Road Directorate, 1996.

[16] Duracrete, Modelling of Degradation. Duracrete, Probabilistic Performance Based Durability Design of Concrete Structures, European Union-Brite EuRam III, 1998.

[17] M. G. Stewart and D. V. Rosowsky, "Time-dependent reliability of deteriorating reinforced concrete bridge decks," Structural Safety, vol. 20, no. 1, pp. 91-109, 1998.

[18] M. G. Stewart, M. H. Faber, and C. Gehlen, "Temporal and spatial aspects of probabilistic corrosion models," in Proceedings of the 3rd International IABMAS Workshop on Life-cYcle Cost Analysis And Design of Civil Infrastructure Systems and The JCSS Workshop on Probabilistic Modelling of Deterioration Processes in Concrete Structures, vol. 2003, pp. 269-278, Lausanne, Switzerland, 2004.

[19] P. Thoft-Christensen, F. M. Jensen, C. R. Middleton, and A. Blackmore, "Assessment of the reliability of concrete slab bridges," in Proceedings 7th IFIP WG 7.5 Working Conference, Reliability and Optimization of Structural Systems, D. M. Frangopol, R. B. Corotis, and R. Rackwitz, Eds., pp. 323-328, 1996.

[20] K. Tuutti, "Corrosion of steel in concrete," CBI Research Report 4-82, Swedish Cement and Concrete Research Institute, 1982.

[21] P. Schie $\beta 1$ and M. Raupach, "Non-destructive permanent monitoring of the corrosion risk of steel in concrete," in NonDestructive Testing in Civil Engineering, vol. 2, pp. 661-674, British Institute of Non-Destructive Testing, Northampton, UK, 1993.

[22] M. Raupach, "Smart Structures: development of sensors to monitor the corrosion risk for the reinforcement of concrete bridges," in Proceedings of the International Conference on 
Bridge Maintenance, Safety and Management (IABMAS '02), J. R. Casas, D. M. Frangopol, and A. S. Nowak, Eds., CIMNE, Barcelona, Spain, 2002.

[23] H. O. Madsen, "Model updating in reliability analysis, Proc," in Proceedings of the 5th International Conference on Applications of Statistics and Probability to Soil and Structural Engineering, pp. 564-577, Vancouver, Canada, 1987.

[24] H. O. Madsen and J. D. Sorensen, "Probability-based optimization of fatigue design, inspection and maintenance," in Proceedings of the Integrity of Offshore Structures, Glasgow, UK, 1990.

[25] R. Zheng and B. R. Ellingwood, "Role of non-destructive evaluation in time-dependent reliability analysis," Structural Safety, vol. 20, no. 4, pp. 325-339, 1998.

[26] T. Onoufriou, "Reliability based inspection planning of offshore structures," Marine Structures, vol. 12, no. 7-8, pp. 521$539,1999$.

[27] T. D. Righiniotis, "Influence of management actions on fatigue reliability of a welded joint," International Journal of Fatigue, vol. 26, no. 3, pp. 231-239, 2004.

[28] M. H. Faber and J. D. Sorensen, "Indicators for inspection and maintenance planning of concrete structures," Structural Safety, vol. 24, no. 2-4, pp. 377-396, 2002.

[29] M. I. Rafiq, M. Chryssanthopoulos, and T. Onoufriou, "Sensitivity of uncertainty in performance prediction of deteriorating concrete structures," Structure and Infrastructure Engineering, vol. 2, no. 2, pp. 117-130, 2006.

[30] K. A. T. Vu and M. G. Stewart, "Structural reliability of concrete bridges including improved chloride-induced corrosion models," Structural Safety, vol. 22, no. 4, pp. 313-333, 2000.

[31] A. Lentz, M. H. Faber, and T. Jonsen, "Halfcell potential measurements for condition assessment," in Proceedings of the International Conference on Bridge Maintenance, Safety and Management (IABMAS '02), J. R. Casas, D. M. Frangopol, and A. S. Nowak, Eds., pp. 365-366, CIMNE, Barcelona, Spain, 2002.

[32] M. K. Chryssanthopoulos and G. Sterrit, "Integration of deterioration modelling and reliability assessment for reinforced concrete bridge structures," in Proceedings of the Asranet International Colloquium, Glasgow, UK, July 2002. 

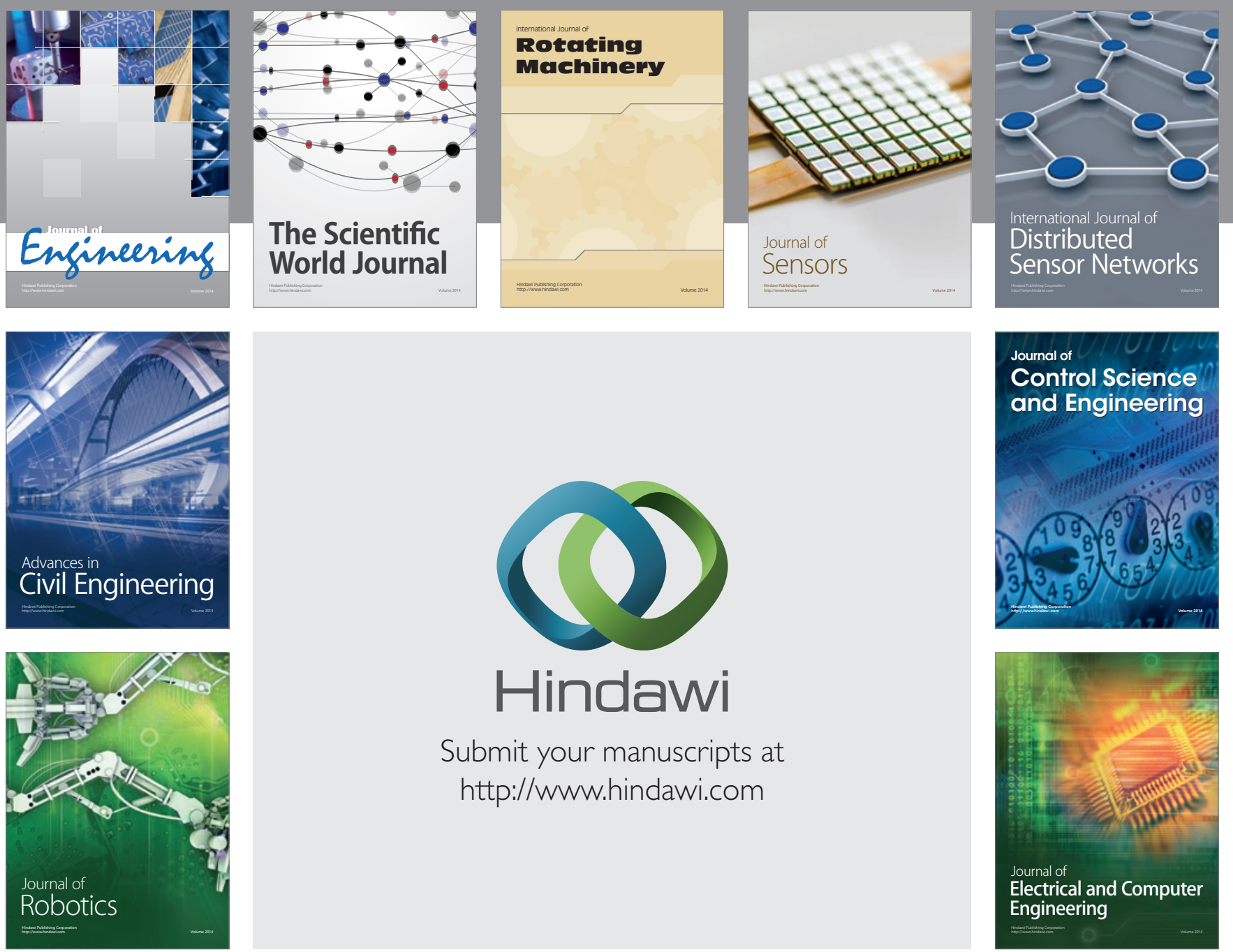

Submit your manuscripts at

http://www.hindawi.com
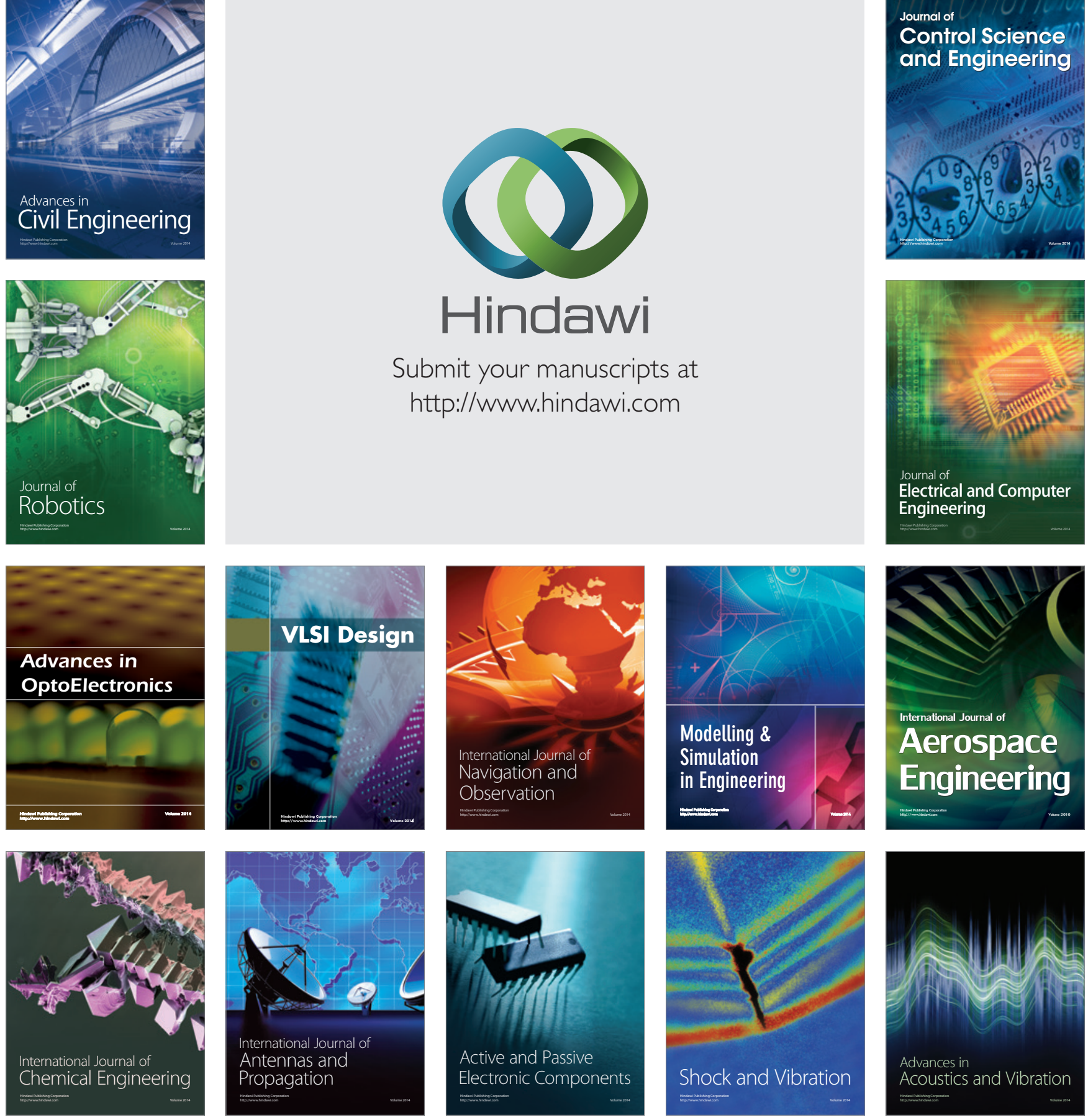